\title{
The Role of Electrophysiologic Study for Prediction and Treatment of Life-threatening Arrhythmias
}

\author{
Harumizu Sakurada, M.D., Takeshi Motomiya, M.D. \\ and Masayasu Hiraoka, M.D**
}

\begin{abstract}
The prognostic significance of drug therapy based on the electrophysiologic study (EPS) was examined during a mean follow-up period of 32 months in 45 patients with sustained ventricular tachycardia (SVT) and in 87 with nonsustained VT (NSVT), and in 7 survivors of cardiac arrest. The drug treatment during the follow-up period was divided into EPS-guided therapy and empirical therapy; in the former therapy, an effective drug for prevention of induced VT by EPS was given and in the latter therapy, an empirical drug was used because there was no effective drugs by EPS. Occurrence of SVT or sudden cardiac death was considered as an arrhythmic event. Of 45 patients with SVT, Group I consisted of 32 cases with organic heart disease (OHD) and Group II, 13 without OHD. In Group I, arrhythmic event occurred in only 2 of 15 patients with EPS-guided therapy, whereas 9 of 13 cases with empirical therapy had arrhythmic event $(\mathrm{p}<0.01)$. In Group II, no arrhythmic event was observed in the 9 patients with EPS-guided therapy, whereas it was seen in 3 of the 4 patients with empirical therapy $(\mathrm{p}<0.05)$. Of 87 patients with NSVT, 61 cases had OHD (Group III). SVT was induced by EPS in 13 patients in Group III. Arrhythmic event was not observed in 8 patients with EPS-guided therapy, whereas it was seen in 3 of the 5 patients with empirical therapy $(p<0.05)$. Arrhythmic event occurred in 2 survivors of cardiac arrest who underwent empirical therapy.

These results suggest that EPS is a useful method for the prediction of lifethreatening arrhythmias and for the selection of optimal drugs to prevent these arrhythmias.
\end{abstract}

$\mathbf{S}$ INCE ventricular tachycardias (VT) are frequently associated with a poor prognosis, the mode of treatment is important in any clinical setting. In 1972 , Wellens et al ${ }^{1}$ first reported that recurrent VT could be reproducibly initiated and terminated by a programmed electrical stimulation of the heart during electrophysiologic study. Since then, the ability to reproduce clinically

Key words:

Life-threatening arrhythmias

Sustained ventricular tachycardia

Nonsustained ventricular tachycardia

Survivors of cardiac arrest

Electrophysiologic study occurring VT in the catheterization laboratory has opened a new approach to diagnose and treat patients suffering from ventricular life-threatening arrhythmias.

While the usefulness of programmed electrical stimulation to assess patients who have a history of sustained VT is well recognized, ${ }^{2-4}$ its role in non-sustained VT has not been clarified:-7 In addition, it has been reported that VT is easily induced with programmed electrical stimulation in patients who have survived cardiac arrest with poor clinical outcomes.

In this study, we carried out electrophysiologic studies (EPS) in patients with VT

The Department of Cardiology, Tokyo Metropolitan Hiroo General Hospital; *Department of Cardiovascular disease, Medical Research Institute, Tokyo Medial and Dental University; Tokyo, Japan

Mailing address: Harumizu Sakurada, M.D., Department of Cardiology, Tokyo Metropolitan Hiroo General Hospital, Ebisu 2-34-10, Shibuya-ku, Tokyo 150, Japan 
and survivors of cardiac arrest. The results of the EPS-guided therapy were evaluated during the follow-up periods in terms of clinical outcomes of the arrhythmic events.

\section{METHOD}

\section{Study patients:}

One hundred and thirty-nine patients, 105 men and 34 women, were studied. These included 45 patients with sustained VT (SVT); 87 with nonsustained VT (NSVT); and 7 survivors of cardiac arrest. Their ages ranged from 16 to 76 years with mean age of $52 \pm 17$. One hundred patients suffered from organic heart diseases; 58 had old myocardial infarction. The left ventricular ejection fraction ranged from 7 to $83 \%$ (average $54 \%$ ). All patients had sufficient cardiac performance to handle routine, daily activity. Patients who had suffered from acute myocardial infarction, acute heart failure, and electrolyte imbalance were excluded from the study.

Definitions. SVT was defined as VT of $\geqq 30$ seconds' duration or requiring termination before 30 seconds because of hemodynamic deterioration.

NSVT was defined as spontaneously terminating VT lasting longer than 6 complexes but $<30$ seconds.

Electrophysiologic study (EPS). All studies were performed with patients in nonsedated, fasting state after all antiarrhythmic drugs had been discontinued at least for 48 hr. Incremental atrial pacing at a rate of 100 beats/ $\mathrm{min}$ to the rate that resulted in 2 nd degree atrioventricular block was performed, followed by programmed atrial stimulation with single atrial depolarizations at one or more drive cycle lengths from the high right atrium.

Right ventricular stimulation was performed at the apex and outflow tract with one to three extrastimuli at two or more drive cycle lengths (most often 600 and 400 msec) and incremental pacing to a minimal cycle length of $250 \mathrm{msec}$. Bipolar stimuli were delivered with a pulse width of 1.5 msec at two times of diastolic threshold strength. Single extrastimuli scanned diastole in $10 \mathrm{msec}$ decrements until SVT was induced or ventricular refractoriness was reached. Double extrastimuli were then del- ivered with the $S_{2}$ placed $50 \mathrm{msec}$ beyond the effective refractory period of the ventricle and the $S_{2}-S_{3}$ interval initially equal to the $\mathrm{S}_{1}-\mathrm{S}_{2}$ interval. Each stimulus was decremented by $10 \mathrm{msec}$ intervals. If double extrastimuli at both the right ventricular apex and outflow tract failed to induced SVT, triple extrastimuli were delivered. The protocol with triple extrastimuli began with $\mathrm{S}_{2}$ placed $50 \mathrm{msec}$ beyond the effective refractory period and the $S_{2}-S_{3}$ and $S_{3}-S_{4}$ intervals equal to the $S_{1}-S_{2}$ interval. Each extrastimulus was decremented by $10 \mathrm{msec}$. Left ventricular stimulation was performed in patients with documented SVT or previous cardiac arrest if no arrhythmias could be initiated by rigth ventricular stimulation.

Electrocardiographic leads $I, a V_{F}$ and $V_{l}$, together with intracardiac electrograms from the atrium, atrioventricular junction, and one or more right ventricular sites, were recorded simultaneously on a multichannel oscilloscope. Intracardiac electrograms were filtered at 30 to $500 \mathrm{~Hz}$. Data were recorded simultaneously on magnetic tape (SONY A-614, Tokyo) and a ink-jet recorder (NIHON-KOHDEN RIJ-2108, Tokyo) at a paper speeds of $100 \mathrm{~mm} / \mathrm{sec}$.

Drug testing. If SVT or NSVT was initiated during control EPS, sequential drug studies were performed. Antiarrhythmic drugs that are currently approved for clinical use in Japan were administerd either orally or intravenously. Selection of drug regimens was based on each patient's history of drug tolerance and previous clinical response. Drugs were tested repeatedly until an effective drug was found. Each drug was given at least 48 hours after the pervious trial.

The test drug was judged effective according to the following findings: for patients with induced SVT during the control, only 15 or fewer complexes of VT were induced after drug administration; for patients with induced NSVT, no VT was induced at all after drug administration.

Follow-up. Assignment of chronic antiarrhythmic therapy was based on the results of electrophysiologic drug testing. When EPS disclosed an effective drug to prevent induction of VT, the patients were given the oral treatment of the effective drug. We designated the above protocol of the treat- 
TABLE I UNDERLYING HEART DISEASES OF STUDIED PATIENTS

\begin{tabular}{|c|c|c|}
\hline \multirow[t]{6}{*}{ Group I } & SVT with $O H D$ & $32 \mathrm{pts}$ \\
\hline & $O M I$ & 17 \\
\hline & $D C M$ & 6 \\
\hline & $A R V D$ & 3 \\
\hline & non-ischemic $L V$ aneurysm & 3 \\
\hline & Others & 3 \\
\hline Group II & $\begin{array}{l}\text { SVT without } O H D \\
\quad(M 11, F 2, \text { age } 35 \pm 21)\end{array}$ & $13 \mathrm{pts}$ \\
\hline \multirow[t]{6}{*}{ Group III } & $\begin{array}{l}\text { NSVT with } O H D \\
\quad(M 44, F 17, \text { age } 54 \pm 14)\end{array}$ & $61 \mathrm{pts}$ \\
\hline & $O M I$ & 39 \\
\hline & $D C M$ & 12 \\
\hline & $H C M$ & 3 \\
\hline & Valvular Disease & 5 \\
\hline & Others & 2 \\
\hline Group IV & $\begin{array}{l}\text { NSVT without } O H D \\
\quad(M 17, F 9, \text { age } 44 \pm 14)\end{array}$ & $26 \mathrm{pts}$ \\
\hline \multirow[t]{5}{*}{ Group V } & $\begin{array}{l}\text { Survivors of Cardiac Arrest } \\
\quad(M 5, F 2 \text {, age } 53 \pm 20)\end{array}$ & $7 p t s$ \\
\hline & $O M I$ & 2 \\
\hline & $D C M$ & 2 \\
\hline & Valvular Disease & 1 \\
\hline & Others & 2 \\
\hline
\end{tabular}

SVT: sustained ventricular tachycardia, NSVT: nonsustained ventricular tachycardia, OHD: organic heart disease, OMI: old myocardial infarction, DCM: dilated cardiomyopathy, ARVD: arrhythmogenic right ventricular dysplasia, HCM: hypertrophic cardiomyopathy

ment as the EPS-guided therapy. The patients who did not respond to any drug testing in EPS were treated with empirical antiarrhythmic drug (the empirical therapy). In 4 cases surgery was performed. The patients in whom programmed ventricular stimulation failed to induce VT were generally not given antiarrhythmic drugs during the observation period.

Clinical follow-up data were obtained at 1- to 6-month intervals. The observation periods ranged from one month to 62 months and an average of 32 months. If occurrence or recurrence of SVT was observed or if a sudden cardiac death occurred, we judged that an arrhythmic event occurred. Sudden cardiac death was defined as an unexpected, witnessed death occuring within $1 \mathrm{~h}$ of the onset of symptoms representing a catastrophic change in the pa- tient's state of health which was unexplainable by any other obvious events. Unwitnessed death was considered a sudden death if: (1) the patient had been seen within 12 hours prior to the death; (2) he had been free of chest pain or any other significant symptoms; (3) the patient's overall clinical status had been stable prior to the fatal event.

Statistical analysis:

Patients were classified into the following 3 categories: patients with a history of SVT, those with NSVT, and survivors of cardiac arrest. Each category of patients was further subclassified according to whether or not they had organic heart disease and divided into 5 Groups (Table I). Then comparisons were made to examine the prognostic differences in the frequency of arrhythmic events among the groups of patients with induced 


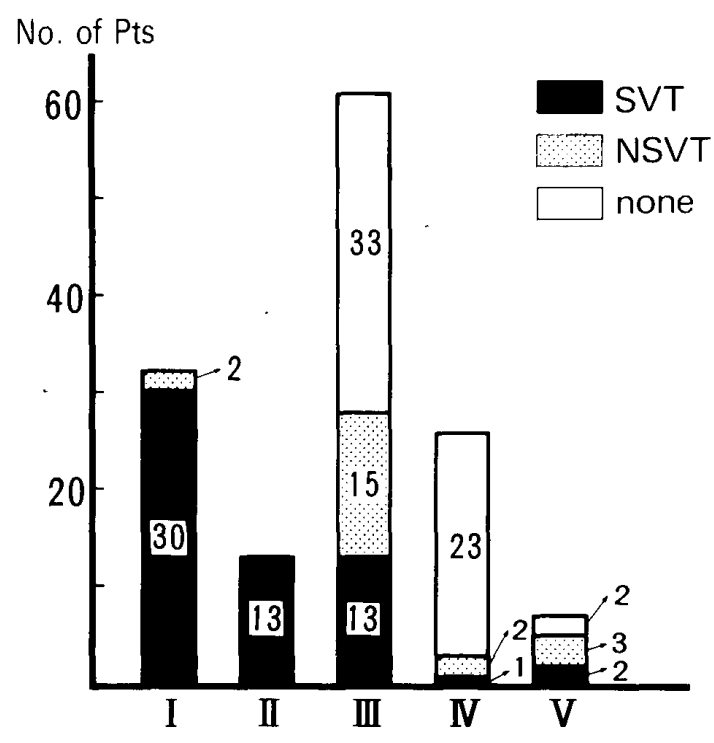

Fig.1. Induction of VT by programmed ventricular stimulation.

VT responsible to the test drug, those for whom no efficacious drug was identified, and those with no inducible VT.

Univariate correlation was tested using chi-square satistic. A probability $\mathrm{p}<0.05$ was accepted as the limit of significance.

\section{RESULTS}

1. Patients with sustained ventricular tachycardia

Table I shows basal condition of heart disease in 45 patients who experienced SVT. Thirty two patients, 28 male and 4 female, had organic heart disease (Group
TABLE II EFFICACY OF ANTIARRHYTHIMIC DRUGS ON INDUCED VT IN PATIENTS WITH SVT

\begin{tabular}{ccc}
\hline \hline Group & $S V T$ & NSVT \\
\hline$I$ & $13 / 30(43 \%)$ & $2 / 2(100 \%)$ \\
\hline$I I$ & $9 / 13(69 \%)$ & \\
\hline
\end{tabular}

I). Their average age was $52 \pm 16$. Organic heart diseases include 17 cases of myocardial infarction, 7 of dilated cardiomyopathy, 3 of arrhythmogenic right ventricular dysplasia, 3 of nonischemic left ventricular aneurysm. There are one case each of cardiac sarcoidosis, hypertrophic cardiomyopathy, and previous surgery for tetralogy of Fallot. Thirteen patients, 11 male and 2 female, had no organic heart disease (Group II). Their average age was $35 \pm 21$.

Figure 1 shows the induction rate of VT in the studied patients. In Group I, programmed ventricular stimulation induced SVT in 30 of 32 patients (94\%), and NSVT in 2. Although two or more different morphologies of SVT were induced in 12 patients, clinical VT which showed identical QRS pattern to that of spontaneous VT was induced in all the patients. In Group II, a single type of clinical VT was induced in all the patients.

Table II summarizes the results of drug testing for patients in whom VT was induced. Among the patients with induced

TABLE III CLINICAL OUTCOME OF PATIENTS WITH SVT

\begin{tabular}{|c|c|c|c|c|}
\hline & & . & \multicolumn{2}{|c|}{$\begin{array}{l}11 \\
13\end{array}$} \\
\hline & \multicolumn{2}{|c|}{ Effective drugs } & \multicolumn{2}{|c|}{ Effective drugs } \\
\hline & +15 & -13 & +9 & -4 \\
\hline Death & 0 & 3 & 0 & 0 \\
\hline$S V T$ & 2 & 6 & 0 & 3 \\
\hline \multirow{3}{*}{$\begin{array}{l}\text { Arrhythmic } \\
\text { event }\end{array}$} & $2 / 15$ & $9 / 13$ & $0 / 9$ & $3 / 4$ \\
\hline & $13 \%$ & $69 \%$ & $0 \%$ & $75 \%$ \\
\hline & \multicolumn{2}{|c|}{$p<0.01$} & \multicolumn{2}{|c|}{$p<0.05$} \\
\hline
\end{tabular}


TABLE IV CLINICAL OUTCOME OF PATIENTS WITH NSVT

\begin{tabular}{|c|c|c|c|c|c|c|}
\hline & \multicolumn{5}{|c|}{ III 61} & \multirow{4}{*}{$I V 26$} \\
\hline & \multirow{2}{*}{\multicolumn{2}{|c|}{$\begin{array}{c}\text { Induced SVT } 13 \\
\text { Effective drugs }\end{array}$}} & \multirow{2}{*}{\multicolumn{2}{|c|}{$\frac{\text { Induced NSVT } 15}{\text { Effective drugs }}$}} & \multirow{3}{*}{$\begin{array}{c}\text { No induction } \\
\text { of VT } 33\end{array}$} & \\
\hline & & & & & & \\
\hline & +8 & -5 & +9 & -6 & & \\
\hline Death & 0 & 2 & 0 & $l$ & 0 & 0 \\
\hline$S V T$ & 0 & 1 & 0 & 0 & 0 & 0 \\
\hline \multirow[t]{3}{*}{ Arrhythmic event } & $0 / 8$ & $3 / 5$ & $0 / 9$ & $1 / 6$ & $0 / 33$ & $0 / 26$ \\
\hline & $0 \%$ & $60 \%$ & $0 \%$ & $17 \%$ & $0 \%$ & $0 \%$ \\
\hline & \multicolumn{2}{|c|}{$p<0.05$} & \multicolumn{2}{|c|}{$N . S$. } & & \\
\hline
\end{tabular}

TABLE $V$ ELECTROPHYSIOLOGICAL FINDINGS AND CLINICAL OUTCOME IN SURVIVORS OF ARREST (N=7)

\begin{tabular}{|c|c|c|c|c|c|}
\hline & \multicolumn{2}{|c|}{ Induced SVT 2} & \multicolumn{2}{|c|}{ Induced NSVT 3} & \multirow{2}{*}{$\begin{array}{c}\text { No induction } \\
\text { of } V T\end{array}$} \\
\hline & \multicolumn{2}{|c|}{ Effective drugs } & \multicolumn{2}{|c|}{ Effective drugs } & \\
\hline & +1 & -1 & +1 & -2 & 2 \\
\hline Death & 0 & 1 & 0 & $I$ & 0 \\
\hline$S V T$ & 0 & 0 & 0 & 0 & 0 \\
\hline Arrhythmic event & $0 / 1$ & $1 / 1$ & $0 / 1$ & $1 / 2$ & $0 / 2$ \\
\hline
\end{tabular}

SVT during EPS, an average of 4.2 different types of antiarrhythmic agents were used for the drug testing. Effective drugs were identified for 13 of 30 patients in Group I (43\%) and 9 of 13 in Group II $(69 \%)$. Effective drugs were also found for 2 patients in Group I with induced NSVT. Four of 17 patients in Group I for whom effective drugs were not identified underwent surgical treatment, because SVT accompanied by hemodynamic deterioration was induced during the drug testing.

Table III shows the clinical outcome of 41 patients who received medical therapy. In 15 patients of Group I, for whom an effective drug was found by EPS, there were no sudden cardiac deaths and two recurrences of SVT during the follow-up period. On the other hand, in 13 patients for whom no effective drug to prevent VT by EPS was found and who underwent empirical drug therapy, sudden cardiac death was observed in 3 patients and recurrence of SVT in 6 . The difference of arrhythmic events between the group of patients, given the EPS-guided drug therapy, and those who received empirical drug was $\mathrm{p}<0.01$. The 3 patients with sudden cardiac death had old myocardial infarction. In 8 patients of Group II who received EPSguided drug therapy, there were no events of sudden cardiac death nor recurrence of SVT, whereas there were recurrence of SVT in 3 of the 4 patients for whom effective drugs were not found and empirical drug therapy was given $(p<0.05)$.

\section{Patients with nonsustained ventricular} tachycardia

Table I also shows the conditions of the heart diseases in patients with documented NSVT in ambulatory ECG. There were 61 
patients, 44 male and 17 female, who had organic heart diseases (Group III). Their average age was $54 \pm 14$. The organic heart diseases include 39 cases of old myocardial infarction, 12 of dilated cardiomyopathy, 3 of hypertrophic cardiomyopathy, 5 of valvular disease, and 2 of other diseases. The number of patients who did not have organic heart diseases (Group IV) was 26 (17 male and 9 female). Their average was $44 \pm 14$.

Figure 1 also shows the induction rate of VT using programmed ventricular stimulation in patients with a history of NSVT. In Group III, SVT was induced in 13 of 61 patients $(21 \%)$. Eleven of these 13 patients had old myocardial infarction and 2 had dilated cardiomyopathy. NSVT was induced in 15 patients $(25 \%)$. Neither SVT nor NSVT was induced in the remaining 33 patients $(54 \%)$. In Group IV, SVT was induced in 1 of 23 patients, and NSVT in 2.

Table IV shows the results of drug testing for the patients with a history of NSVT and their clinical outcomes. A mean of 3.2 different types of antiarrhythmic drugs were used in the testing. Effective drugs were found for 8 of the 13 patients with induced SVT by EPS. Therefore, the 8 patients underwent EPS-guided drug therapy. No arrhythmic events occurred in any of them during the follow-up period. However, in the 5 patients who received empirical drug treatment during the follow-up period, 2 had sudden cardiac death and 1 had new occurrence of SVT. The difference in terms of occurrence of arrhythmic event between the patients with and those without effective drugs was significant $(\mathrm{p}<0.05)$. The 2 patients with sudden cardiac death had old myocardial infarction and 1 patient with SVT had dilated cardiomyopathy.

Of the 15 patients in whom programmed ventricular stimulation induced NSVT, effective drugs were identified in 9 , all of whom had good prognosis, whereas sudden cardiac death was observed in 1 of the 6 patients for whom effective drugs were not identified. No difference in the occurrence of arrhythmic events between patients with effective drugs identified and those without, were found among patients in whom NSVT was induced. The remaining 33 patients in Group III who failed induction of VT by programmed ventricular stimulation had good prognosis showing no arrhythmic events during the follow-up period.

In Group IV, no arrhythmic event was observed in any of the patients regardless of whether VT was induced or not, and whether effective drugs were identified or not.

\section{Survivors of cardiac arrest}

Seven patients, 5 male and 2 female, survived cardiac arrest. Their average age was $53 \pm 20$. All of them had organic heart disease, as is shown in Table I. Table V shows the relationship between the results of drug testing by EPS in the survivors of cardiac arrest and their clinical outcome. Of 7 patients, SVT was induced in 2, NSVT was induced in 3 and no VT was induced in the remaining 2. During the observation period, sudden cardiac death occurred in 2 cases. The clinical diagnoses of those patients were myocardial infarction and dilated cardiomyopathy. Effective drugs ware not identified for either of them.

\section{DISCUSSION}

The present study demonstrated that the patients who were receiving an effective drug for the prevention of induced VT by electrophysiologic study (EPS) had a low recurrence rate of life-threatening arrhythmias compared th those for whom no effective drug was found by EPS, and were taking an empirical antiarrhythmic agent. Therefore, two factors seem to be important for determining prognosis during the follow-up period of patients who are clinically at risk of life-threatening arrhythmias; one factor is to reproduce either clinical VT or SVT by the programmed ventricular stimulation in EPS, and another is to find an effective drug for prevention of these induced VT.

VT is important clinically for two reaons: one is to cause a rapid hemodynamic deterioration with the onset of the arrhythmia, especially in cases with organic heart disease, and the other is to become a precursor of fatal arrhythmias such as ventricular fibrillation or flutter. A study for analysis of ambulatory ECG recordings in patients suffering from sudden cardiac death showed that approximately $80 \%$, of the cases had 
ventricular fibrillation which, in many cases, was preceded by $\mathrm{VT}^{11}$. Therefore, it is of prime importance to prevent an occurrence of VT in patients who have a documented or suspected history of ventricular tachyarrhythmias and sudden cardiac arrest.

Various reports indicate that patients with SVT are likely to experience a recurrence of the same type of VT or sudden cardiac death, unless appropriate therapy is given?-4 In the case of NSVT, however, no definite conclusion has been reached as to whether or not NSVT itself can be a precursor of life-threatening arrhythmias, or what types of patients should undergo therapy, and how these patients could be differentiated from those with a good prognosis:-7

We examined the significance of electrophysiologic study in the prediction of clinical prognosis of life-threatening arrhythmic events in patients with a clinically identified SVT, those with NSVT recorded with ambulatory ECG, and survivors of cardiac arrest. It was found, as other researchers had reported ${ }^{2-4}$ that programmed ventricular stimulation easily induced SVT showing identical QRS morphologies to those documented clinically and that the use of antiarrhythmic drugs to prevent induction of VT significantly reduced the occurrence of arrhythmic events. It was also revealed that patients with organic heart diseases (especially old myocardial infarction), for whom no effective drugs had been identified, were likely to suffer from suden cardiac death during the follow-up period. This leads us to the conclusion that for the patients having organic heart disease and SVT for whom no effective drugs could be identified in the drug testing by EPS using programmed ventricular stimulation, we should consider the use of surgical ablation, catheter ablation, an automatic implatable cardioverter defibrillator, or new drugs under evaluation such as amiodarone! $!^{2-15}$

While the patients with a history of SVT had a high induction rate of the arrhythmia by programmed ventricular stimulation, the induction rate of SVT in cases with NSVT was low. It was also indicated that the induction rate of SVT was extremely low for cases without any discernible organic heart diseases. This difference is probably attributed to the fact that the mechanism of most SVT is re-entry while various mechanisms may be involved in the generation of NSVT.

In this study, SVT was induced in $21 \%$ of the patients with NSVT having organic heart diseases. Arrhythmic events occurred more frequently in patients for whom no effective antiarrhythmic drugs were identified in the drug testing using the programmed ventricular stimulation than in patients for whom such drugs could be identified or those in whom SVT was not induced. This significant differnce in the number of arrhythmic events between the 2 groups of patients indicates the usefulness of EPS and programmed stimulation in predicting lifethreatening arrhythmias and in selection of an efficient therapy for the patients displaying NSVT by ambulatory ECG monitoring.

The study also showed that patients with NSVT, in whom programmed ventricular stimulation failed to induce VT, had good prognosis for arrhythmic event even without administration of an antiarrhythmic drug. Moreover, programmed ventricular stimulation could only induce VT in a few patients showing NSVT without organic heart disease. Therfore, it may be safe to conclude that patients showing NSVT, but having no organic heart disease, carry a good prognosis for arrhythmic event. For this reason, EPS is not indicated for patients in this group.

All of the survivors of cardiac arrest had organic heart disease. Programmed ventricular stimulation induced NSVT in $43 \%$, and SVT in $29 \%$ of these patients. Two patients who suffered sudden cardiac death during the follow-up period were those in whom VT could be induced without identification of effective antiarrhythmic drugs. This observation was in line with previous reports dealing with patients who recovered from cardiac arrest 8,9 This is another indication that EPS for survivors of cardiac arrest is useful to predict and treat the recurrence of arrhythmic events.

We conclude that the electrophisologic study in patients with sustained ventricular tachycardia and nonsustained ventricular tachycardia based on organic heart disease, and those with a history of cardiac arrest provides important information for predicting life-theatening arrhythmias as well as for 
selectiong an appropriate therapy.

\section{REFERENCES}

1. WELLENS HJJ, SCHUILENBURG RM, DURRER D: Electrical stimulation of the heart in patients with ventricular tachycardia. Circulation 46: 216,1972

2. HOROWITZ LN, JOSEPHSON ME, FARSHIDI A, SPIELMAN SR, MICHELSON EL, GREENSPAN AM: Recurrent sustained ventricular tachycardia: role of the electrophysiologic study in selection of antiarrhythmic regimens. Circulation 58: 986,1978

3. MASON JM, WINKLE RA: Accuracy of the ventricular tachycardia induction study for predicting long-term efficacy and inefficacy of antiarrhythmic drugs. $N$ Eng $J$ Med 303: 1073,1980

4. RAE AP, GREENSPAN AM, SPIELMAN SR, SOKOLOFF NM, WEBB CR, KAY HR, HOROWITZ LN: Antiarrhythmic drug efficacy for ventricular tachyarrhythmias assosiated with coronary artery diseases as assesed by electrophysiologic studies. Am J Cardiol 55: 1494, 1985

5. BUXTON AE, WAXMANN HL, MARCHLINSKI FE, JOSEPHSON ME: Electrophysiologic studies in nonsustained ventricular tachycardia: relation to underlying heart disease. Am J Cardiol 52: 985,1983

6. VELTRI EP, PLATIA EV, GRIFFITH LSC, REID PR: Programmed electrical stimulation and long term follow-up in asymptomatic nonsustained ventricular tachycardia. Am J Cariol 56: 309, 1985

7. SULPIZI AM, FRIEHLING TD, KOWEY PR: Value of electrophysiologic testing in patients with nonsuatained ventricular tachycardia. Am J Cardiol 59: 841,1987

8. FREEDMAN RA, SWERDLOW CD, SODERHOLM-DIFATTE V, MASON JW: Prognostic significance of arrhythmia inducibility or noninducibility at initial electrophysiologic study in survivors of cardiac arrest. Am J Cardiol 61: 578,1988

9. WILBER DJ, GARAN H, FINKELSTEIN D: Out-of-hospital cardiac arrest: use of electrophysiologic testing in the prediction of long-term outcome. N Engl J Med 318: 19, 1988

10. ROY D, WAXMAN HL, KIENZLE MG, BUXTON AE, MARCHLINSKI FE, JOSEPHSON ME: Clinical characteristics and long-term followup in 119 survivors of cardiac arrest: relation to inducibility at electrophysiologic testing. $A m J$ Cardiol 52: 969, 1983

11. PANDIS IP, MORGANROTH J: Holter monitoring and sudden cardiac death. Cardiovascular Reviews and Reports 5: 283, 1984

12. MILLER JM, KIENZLE MG, HARKEN AH, JOSEPHSON ME: Sbendocardial resection for ventricular tachyvardia: predictors of surgical success. Circulation 70: 624, 1984

13. HARTZLER GO: Electrode catheter ablation of refractory focal ventricular tachycardia. $J \mathrm{Am}$ Coll Cardiol 2: 1107, 1983

14. MIROWSKI M: The automatic implantable cardiovrter defibrillator: an overview. J Am Coll Cardiol 6: 461, 1985

15. HAMER AW, FINERMAN WB, PETER T, MANDEL W: Disparity between the clinical and electrophysiologic effects of amiodarone in the treatment of recurrent ventricular tachycardia. Am Heart J 102: 992, 1981 